\title{
A PROPÓSITO DEL BICENTENARIO: UNA REVISIÓN DE CENTROAMÉRICA COMO ESPACIO-RUTA DESDE LA GEOPOLIITICA CRÍTICA
}

\author{
REGARDING THE BICENTENARY: A REVIEW OF \\ CENTRAL AMERICA AS A SPACE-ROUTE \\ FROM CRITICAL GEOPOLITICS
}

\author{
Karen Arévalo Mejía ${ }^{1}$ \\ ORCID: 0000-0002-3107-2387
}

\section{RESUMEN}

Los procesos independentistas en Centroamérica se han visto influenciados por su ubicación de gran importancia geopolítica dentro del continente, la cual ha servido de espacio-ruta para las potencias, especialmente desde los años ochenta hasta la actualidad. Esto también ha intervenido en la conformación de los Estados del istmo, fragmentando su desarrollo y debilitando su po-tencial, lo que expone a la región, aún más, a los intereses de poderes externos.

De esta forma, analizar Centroamérica desde la geopolítica crítica permitirá su entendimiento como poseedora de espacios de poder y cómo estos han sido tomados por potencias para ejercer dominio sobre la zona, interfiriendo, incluso, con las opciones de integración. Su localización, en el nivel general, ha servido como una ruta de comercio internacional de gran trascendencia, una de las razones de la disputa geopolítica por el control del Canal de Panamá. Además, en esta condición de paso, queda abierta la posibilidad de otros flujos, como es el caso de las personas migrantes o del tráfico de drogas.

La región centroamericana se ha construido bajo directrices externas y desde arriba, por lo

\section{ABSTRACT}

The independence processes in Central America have been influenced by the area's geographical position and its geopolitical relevance in the continent. The region has been seen by the global powers as a space-route, especially from the eighties to the present. This geopolitical condition has predisposed the formation of the Central American countries by fragmenting their development process and weakening their whole potential; as a result, the territory is even more vulnerable to external interests. Therefore, analyzing Central America from the Critical Geopolitics perspective will contribute to understand it as a place defined by politics of space in which global powers interfere undermining possibilities for regional integration among other effects.

The geographical location of this region has been used as a trade-route for international transportation, being this one of the reasons for the dispute regarding the Panama Canal's control by global powers; besides its strategic location allows other kinds of flows as it is the case of migrants and drugs, for example. Since the area has been built due to external influences,

1 Universidad de San Carlos de Guatemala (USAC). Profesora y coordinadora. Área de Estudios Internacionales, Instituto de Investigaciones Políticas y Sociales. Licenciada en relaciones internacionales (USAC). Correo electrónico: karenjohis@gmail.com 
que repensarla desde una visión deconstructiva se hace necesario para darle un giro a su rumbo $\mathrm{y}$ aprovechar las fortalezas del istmo, principalmente, para lo interno y, en específico, para su población.

Palabra claves: Bicentenario; Centroamérica; deconstrucción; geopolítica crítica; potencias; ruta internacional. a deconstructive approach is needed to better understand its current reality, thinking about possibilities of positive changes, and taking advantage of its own strengths to benefit its population.

Key words: Bicentenary; Central America; Critical Geopolitics; deconstruction; global powers; international route.

\section{Introducción}

En 1823, Centroamérica se reviste con un doble propósito a partir de la instauración de la República Federal de Centro América, que inicialmente correspondía a designarla como región, pero también como proyecto político. En este sentido, Granados (1986) señala que "tras la independencia, la concepción de Centroamérica como puente y como istmo estaba latente, así como su destino geopolítico" (p. 1).

Es importante resaltar que, pese a que los estudios de Centroamérica se han abordado desde hace mucho tiempo, el interés en la significación del elemento geopolítico en los más connotados análisis centroamericanos ha sido muy poco visibilizado. Generalmente, estos se han trabajado desde otras disciplinas y temáticas, como la historia o la ciencia política, al igual que desde la perspectiva de la integración centroamericana, entre otras. Por ello, en las relaciones internacionales, surge esta propuesta para entender la región, partiendo de esas fuerzas y poderes que, históricamente, han acompañado y condicionado a sus países en conjunto.

A lo largo de la historia centroamericana, se han reflejado acciones, por parte de las potencias, que muestran los intereses geopolíticos como verdaderos agentes, en la mayoría de casos, determinantes y que no solo condicionan los procesos económicos y sociales. Es así como el presente artículo surge con el interés de tratar el bicentenario de independencia centroamericana desde una mirada de la geopolítica crítica, entendiendo la importancia de la región en cuanto a un espacio-ruta que, a lo largo de los años, se ha visto como un escenario (tablero geopolítico) en el cual las potencias han dictado sus líneas y establecido sus intereses.

Para ello, el documento se estructura de la siguiente manera: primero, un abordaje teórico-metodológico orientado a comprender la geopolítica crítica y sus 
bases, así como el concepto de geopolítica y su evolución a lo largo de los años. Seguidamente, se analizan aquellos factores internos y externos que han influido en la historia, al igual que acciones realizadas por los países centroamericanos, las cuales inciden en que las potencias coloquen su mirada en la región. Por último, se reflexiona sobre la posibilidad de la deconstrucción del territorio centroamericano, lo que permitiría definir y repensar una propuesta de una agenda de investigación y abordaje desde la geopolítica crítica y la decolonialidad, a propósito de la conmemoración de doscientos años de vida independiente.

\section{Metodología}

El estudio de los procesos de independencia de Centroamérica se ha desarrollado desde distintas perspectivas teóricas y metodológicas. Muchas de estas investigaciones se han realizado partiendo de diversas conceptualizaciones abordadas desde el pasado colonial, desde el cual se han desprendido estrategias políticas y corrientes que son contrapuestas y divergentes. Así lo señala Palerm \& Wolf (2008), al decir que "de la diversidad de las corrientes políticas, que expresan la conciencia y los intereses de grupos sociales determinados, se derivan ciertas interpretaciones del pasado" (p. 150).

Metodológicamente, este artículo se llevó a cabo en dos etapas orientadas al análisis y a la reflexión del bicentenario de la región centroamericana, desde la mirada de la geopolítica crítica. En la primera fase, se privilegió el examen documental con recursos historiográficos extraídos de Internet; se utilizó el método histórico para vincular distintos periodos en su sucesión cronológica; además, se elaboraron instrumentos como una guía de preguntas y se hizo un conversatorio con historiadores guatemaltecos titulado "Comprendiendo la región Centroamericana desde la geopolítica, a partir de una mirada histórica", para recabar datos, profundizar en aquellos que se pueden abordar desde la historia y, con ello, posteriormente, realizar el análisis desde las relaciones internacionales.

En la segunda fase, se procedió a la sistematización de los datos, así como a contrastar la información obtenida en el webinario "Reflexiones desde la geopolítica sobre Centroamérica en el marco del bicentenario", organizado por la presente Revista de Relaciones Internacionales, en el que la suscrita disertó sobre la posición geopolítica de la región. Todo lo anterior visto desde la luz de la teoría del imperialismo y de la geopolítica crítica. 


\section{Marco teórico}

Como una línea base para el abordaje de esta investigación, es importante reflexionar en torno al significado de Centroamérica. Para ello, es imprescindible retomar lo que rezaba el decreto que dio vida a la Federación Centroamericana en 1823:

El escudo de armas de las Provincias Unidas será un triángulo equilátero. En su base aparecerá la cordillera de cinco volcanes colocados sobre un terreno que se figura bañado por ambos mares; en la parte superior un arco iris que los cubre y bajo el arco el gorro de la libertad esparciendo luces. En el triángulo y en forma circular se escribirá con letras de oro: Provincias Unidas del Centro de América (Granados, 1986, p. 1).

En esta descripción que presenta el autor Carlos Granados, se aprecia la doble definición de Centroamérica; la primera haciendo referencia al nombre, utilizado por primera vez, que refleja el espacio geográfico como un territorio continuo, ubicado en medio de la región norte y sur de América. Por otro lado, se alude al escudo de armas, en el que se visualiza una línea angosta y larga (denominada cordillera), recubierta por dos océanos. Se interpreta Centroamérica como puente entre dos regiones, pero también como istmo; además, se propone otra visión, desde el sentir de los próceres de la independencia, con la cual la región es vista como un eje de la comunicación entre las Américas (norte y sur), como el punto de contacto entre, o bien como paso interoceánico del transporte mundial y de la comunicación.

En un primer momento, Centroamérica es concebida "como puente o zona de contacto" Granados (1986) establece que entre las culturas del continente, tanto del norte como del sur, hasta la llegada del conquistador europeo a la región americana (p. 1). Posteriormente, el rol que juega la zona se redefine, en función de sus principales necesidades de la época, en particular, la comunicación entre los polos políticos económicos, que ya no solo estaban conectados por vía terrestre, sino igual por la vía marítima. Desde la perspectiva de las élites y fundadores de la Federación Centroamericana, el área era estratégica, dada su posición en el intercambio mundial. A partir de este momento, se perfila, prematuramente, su destino geopolítico.

Desde la etapa colonial, seguida por los procesos independentistas, las líneas geopolíticas para Centroamérica han sido trazadas desde afuera, principalmente desde las potencias; en este caso, se hará referencia a aquellas que han tenido más influencia en la región: Estados Unidos, China, Rusia, Reino Unido y España, que en su conjunto se denominarán comunidad internacional (para 
efectos del presente artículo). Dado que el proyecto centroamericano suponía designar como región, pero también como proyecto político, a los países, es necesario reflexionar y entender el camino del territorio centroamericano desde la mirada de la geopolítica crítica y, en este sentido, destaca la necesidad de revisar los aportes de varios autores quienes han contribuido al concepto y a la propia teoría.

Se puede iniciar con John Agnew, el cual asevera que la geopolítica crítica consiste en "el estudio de la deconstrucción de la forma de ver el mundo que va a definir el escenario de la política internacional” (Agnew, 1998, p. 15). Ese escenario mundial se describe como incierto, complejo y distinto a lo establecido, situación que se ve reflejada en la región a partir de los procesos independentistas.

Es importante mencionar que "desde la geopolítica crítica sobresalen elementos como: alinear, conducir, manipular y direccionar la conducta social", que Michael Foucault (2002) denominó "microespacios" de poder (p. 134). Estos elementos clave contribuyen con la comprensión del área centroamericana como un microespacio de poder, asociado tanto a la importancia de la ubicación territorial de Centroamérica como a lo que representa en el imaginario de la política internacional, puesto que la geopolítica crítica permite analizar cómo esta ha sido conducida y direccionada en el escenario internacional, al igual que lo relativo a los intereses y amenazas de otros Estados en la región.

En tal sentido, dichos elementos claves se retoman con base en que Foucault consideraba la geopolítica crítica como un factor y un espacio tanto de reconstrucción como de la transformación global, privilegiando a quienes carecen de poder. De esa cuenta, Centroamérica se convierte en el escenario en el cual se dan todas las mutaciones y buena parte de ellas es conducida o manipulada por parte de las líneas dictadas por las potencias del momento. En estos microespacios de poder, se establecen formas de control, sin embargo, también de resistencia, frente a las instituciones y el aparato tradicional del Estado, que, asimismo, permiten comprender con mayor profundidad las líneas dictadas por parte de la comunidad internacional para la región.

Entre el análisis de la geopolítica crítica, igualmente se les otorga gran importancia a los discursos, en particular porque estos direccionan la conducta social, $\mathrm{y}$, en este caso, justo los discursos de "independencia" provenientes de las élites mismas que han dominado en la construcción de la historia conocida en la zona. 


\section{Sobre el concepto de geopolítica}

La "geopolítica" es un término conveniente de aclarar, debido a sus múltiples cambios a lo largo de los años y porque tiende a ser aplicado al campo militar. En el nivel internacional, el abordaje de la geopolítica estaba dominado por la corriente determinista, integrada por Ritter, con su obra Geografía general comparada (2001); Humboldt, en Ensayo Político sobre el Reino de la Nueva España (1827); Ratzel, en Geografía Política (1895); Kjellen, en El estado como forma de vida (1916), y Haushofer, en Geopolítica del Océano Pacífico (1924). Todos ellos generaron un criterio de crecimiento territorial, fundado en el estatismo nacionalista y expansionista. Estas aproximaciones consideraban al Estado como un organismo vivo y con una dinámica espacial expansiva desde el centro (heartland) a la periferia (rimland).

También, se han reconfigurado nuevos enfoques de estudio de la geopolítica que han incidido en propuestas alternativas de punto de vista, como la geopolítica crítica (algunos de sus estudiosos son Michael Foucault y Johan Galtung)y la geopolítica posmoderna (se hace referencia a David Slater). De acuerdo con González (2017),

en el caso de la geopolítica crítica, se inspira en los trabajos sobre el análisis y la deconstrucción de los discursos de Foucault y Derrida, esta fue, en un inicio, propuesta por Dalby (1991), Agnew (1992) y Ó Tuathail (1998) y surge como una alternativa para la producción de conocimientos geopolíticos a partir de los significados (explícitos e implícitos) conferidos a los lugares en función de la política exterior. (pp.87-88)

Entre los principales indicios de dicha geopolítica, se resalta "los órdenes geográficos, los cuales son creados por actores clave y, a su vez, estos son trasladados a otros escenarios por lo que se puede establecer que la organización del espacio no es una práctica neutral ni objetiva" (Warf, 2006, p. 85). En consecuencia, estos ordenes identifican la complejidad de las sociedades y de los territorios, buscando dar respuesta y explicar de qué manera "el conocimiento del espacio -y su vinculación con el tiempo- es reducido a commodities de seguridad y lugares que necesitan ser controlados, invadidos o domesticados" (Ó Tuathail y Agnew, 1992, p. 195, citado en González, 2017).

Cabe resaltar que, si bien la conceptualización de la geopolítica ha ido evolucionando, está estrechamente ligada a las circunstancias geográficas, que incluyen accidentes en la geografía, la población y los recursos naturales que posea un espacio, pero, incluso, va a actuar sobre factores menos tangibles como la economía y las finanzas. Para Baños (2017), estos elementos son predominantes, 
dado el proceso en el cual se establecen las directrices geopolíticas, puesto que son el "qué", determinadas como necesidad; de igual manera, los intereses del Estado son los "para qué", de los cuales surgirán las estrategias pertinentes convertidas en geoestrategias que son el "cómo" y el "con qué" se llevan a la práctica en las tácticas para alcanzar los fines geopolíticos $\mathrm{y}$, en este sentido, aplicado a la investigación, el abordaje desde la geopolítica crítica.

Los principios descritos anteriormente han prevalecido, de alguna forma, con el paso del tiempo; de acuerdo con Baños (2017) "estos principios inmutables siguen rigiendo las relaciones internacionales y los asuntos del mundo al que pertenecemos" (p. 33).

\section{Centroamérica: desde los factores externos a los internos}

La región centroamericana ha tenido una posición privilegiada en el continente americano, la cual le ha traído luces y sombras que han marcado su historia. Su ubicación le permite conectar el océano Atlántico con el Pacífico y servir de puente entre la región norte y la sur. Desde la llegada de los españoles, Centroamérica se convirtió en una zona que facilitó las rutas de comercio y el control de esta desembocó en los intereses de otros países que llegaron al continente en los años siguientes.

Desde los albores de la formación de las repúblicas independientes y en el proceso de balcanización que siguió al fin del dominio colonial español, en el primer cuarto del Siglo XIX, la sombra de ese factor geopolítico se hizo sentir en los destinos de los pueblos. En adelante y hasta la actualidad, la historia de cada nación del istmo ha estado influida, muchas veces de manera decisiva, por factores externos (CSUCA, 2014, p. 206).

La localización geoestratégica del istmo implicó que potencias como España, Portugal, Francia, Reino Unido y Estados Unidos fijaran sus intereses en él, tanto por su ubicación (que, en general, ha servido como una ruta de comercio facilitadora del transporte, una de las razones en la disputa por el control del Canal de Panamá, por cierto) como por ser utilizada de ruta para el narcotráfico y el paso de personas migrantes, en la actualidad, .

Es importante destacar que Centroamérica siempre se ha caracterizado por tener un gran valor geopolítico y, en aquella época, cumplía un rol estrictamente de carácter defensivo, que implicaba el control de la comunicación entre los océanos, buscando la prosperidad nacional. En por ello que se hace relevante 
mencionar a Mahan (1991), dado que, en su obra The Panama Canal from a Military Point of View, afirmó que "El valor del canal no era su impenetrabilidad como posición, sino su utilidad a la marina como agresor-defensor de toda la línea costera nacional" (citado en Barrios, 2014, p. 201).

En este sentido, esa órbita defensivo-ofensiva y la ubicación centroamericana se convierten en elementos importantes, pues posibilitaban una ágil comunicación, acortando el paso interoceánico. Tal como se menciona en la obra citada de Mahan, "[Con esta región], Centroamérica, se torna fácil lo que es imposible por tierra y da el control de la región del Caribe. La comunicación de la flota norteamericana se realiza mejor... las comunicaciones son probablemente el elemento más vital y determinante en la estrategia militar o naval" (citado en Barrios, 2014, p. 201).

Sobre el significado del Canal, Barrios (2014) menciona: "era el punto vital del sistema de transferencia mediante el cual la Marina de los Estados Unidos podría apoyar la defensa en cualquiera de sus costas, pues las comunicaciones dominaban la guerra en todos sus aspectos" (p.199). Por tal razón, el objetivo principal del Canal de Panamá también cumpliría una doble función, pensando en términos comerciales y militares. Posteriormente, en la década de los ochenta, el espacio centroamericano adquiere una sobredimensionada importancia geopolítica, la cual dio lugar a los actores y sucesos con una valoración exagerada; al respecto, Barrios (2014) argumenta que "parecía como si dentro del istmo se jugara una partida decisiva del enfrentamiento Este-Oeste” (p. 199).

Desde una perspectiva más global, se puede decir que se presenta una Centroamérica que no es homogénea desde sus procesos emancipatorios, hecho que ha ido debilitando a la región y enraizando su dependencia hacia otras potencias. Si bien es cierto la zona se localiza en una posición altamente codiciada, tanto por su valor comercial como por el relativo a seguridad, a lo interno presenta debilidades, particularmente, por su exacerbada heterogeneidad.

Es importante resaltar que parte de ese valor en materia de seguridad que posee la región se puede ejemplificar con las políticas dictadas para sus países, a partir del atentado a las Torres Gemelas. Por ello, en el 2001 el enfoque de seguridad en el área cambió drásticamente; Estados Unidos cambió sus políticas, perfilando a la persona migrante como terrorista y asociando la presencia de terceras naciones como amenazas para la seguridad internacional.

El evento mencionado marcó un hito en la migración y en la seguridad fronteriza, puesto que, desde este, Centroamérica se ubicó como un punto vital en el cual se externalizaron fronteras y se implementaron programas de seguridad 
para el control de la migración; asimismo, se disminuyó el tráfico de migrantes y el narcotráfico hacia el norte del continente. Un ejemplo de lo anterior es el Informe sobre Estrategia Internacional de Control de Narcóticos de 2016, el cual reveló que "el 90\% de los cargamentos de la droga cruzaron primero por América Central antes de llegar a EE.UU” (United States Department of State, 2016), por lo cual se debe comprender la importancia de la zona no solo para los Estados (potencias), sino también para los grupos criminales, como punto estratégico para sus operaciones que conecta las regiones del continente, favoreciendo intereses de diversidad de actores externos.

Para ilustrar dichos intereses, vale mencionar el caso de China. Si bien es cierto a partir del 2018 estrechó su relación con América Latina, especialmente con Centroamérica:

Su nivel de préstamos oficiales se redujo en los dos últimos años dado que Pekín desarrolló otras estrategias en la región y mejoró su posición estratégica en América Central. En este acercamiento, Panamá ha sido la pieza fundamental en el interés de las autoridades chinas para extender el trazado marítimo de la Nueva Ruta de la Seda en Latinoamérica (Villacorta, 2019).

Como bien se conoce, en el recorrido de la historia de Centroamérica, existen factores externos que han incidido con mayor fuerza y eficacia en su transformación después de la independencia y que a la fecha se mantienen. Tal fue el rol de las colonias y las potencias colonizadoras, actores de gran relevancia en la creación del capitalismo.

Se llega, entonces, a que la comprensión de las diversas crisis económicas, sociales y políticas que han afectado a Centroamérica implica la identificación de los actores y factores externos, así como las llamadas "fuerzas internacionales (viejas y nuevas)", que en este análisis se denominan potencias: España, Gran Bretaña y Estados Unidos. Ellas representan una gran influencia interna en la región $\mathrm{y}$, en consecuencia, se han convertido en actores igualmente decisivos en el desarrollo y la eventual solución de los conflictos. Como algunas pocas regiones del planeta, "Centroamérica ha sido objeto de la política exterior, especialmente de los Estados Unidos” (Torres-Rivas, 1985, p. 43).

Reflejo de la presencia del invocado vecino país del norte en los asuntos internos de las naciones, precisamente, son las crisis internas que se han desarrollado en las sociedades centroamericanas y cuyas soluciones han evidenciado, de una u otra forma, la voluntad de la potencia. Como señala Edelberto Torres Rivas (1985), “Centroamérica es el eslabón más débil, lugar en donde las contradicciones de los sistemas internacionales e imperialistas se han llevado a cabo, 
partiendo de la Independencia de Centroamérica que fue sometida al antojo de intereses mexicanos, británicos, españoles y, a su vez, por el miedo de que la región sirviera de puerto para contraataque" (p.52).

Por otro lado, Torres Rivas (1985) indica que esta dinámica internacional de la crisis interna está caracterizada por "un conjunto de relaciones e intereses que se manifiestan de diversa manera, desde la ayuda material en dinero y armas, el reconocimiento diplomático, presiones políticas, apoyo moral y otras formas de solidaridad o rechazo más de naturaleza propagandística"(p.55).

Las manifestaciones de intereses en la región centroamericana han sido evidenciadas en dos expresiones, tal como lo menciona Rivas (1985): 1) con la política de Estados Unidos, que ha buscado insertarse en el área, influyendo y buscando el apoyo de los gobiernos con visiones tradicionales del panamericanismo; 2) en contraste, con la perspectiva de contención y rechazo a la voluntad norteamericana, visible en la política de otros gobiernos y organizaciones internacionales que expresan, con mayor o menor decisión, la política norteamericana para Centroamérica.

Es así que se llega a la conclusión de que con el afán de entender la región centroamericana y conocer su importancia geoestratégica para la comunidad internacional, deben incluirse en su análisis factores externos e internos, pues estos están intrínsecamente relacionados.

Entre los factores internos que se pueden mencionar, se resalta que "la región centroamericana se encontró inmersa en una serie de dinámicas complejas que dificultaron su cohesión y generaron dudas sobre su viabilidad" (Molina, 2019, p. 27). Estas incertidumbres y tensiones en la búsqueda de conformar una unificación del área centroamericana eran reforzadas por el choque entre prácticas de un pasado colonial, el cual fue dirigido por lineamientos hispánicos en contraste con las ideas y los movimientos cuyo objetivo fue gestar un nuevo proyecto de Estado. A lo anterior se agrega que, a lo interno, las discordias y la falta de una visión unificada, debido a la fragilidad política, económica y social de los Estados centroamericanos, conllevaron al rechazo de la hegemonía de Guatemala como líder de la región y, ante ello, se valoró la anexión a otro Estado en el proceso posindependentista, lo cual desencadenó otra serie de enfrentamientos e intereses.

Ese otro Estado que se refiere en ese entonces es el Imperio mexicano, el cual surge como actor en el proceso centroamericano y se vuelve parte de la historia del territorio, tras la invitación que Iturbide realizó a las provincias a unirse a este imperio, incluso meses antes de que se declarara formalmente 
la independencia de España. Jonathan Ordóñez (comunicación personal, 20 de agosto, 2020) señala que, el historiador Mario Vázquez Oliveira, en su reciente obra "El Imperio Mexicano y el Reino de Guatemala: Proyecto Político y Campaña Militar, 1821-1823”, hace un recordatorio de que la relación de Centroamérica con México comienza con dicha invitación, la cual traía de fondo la idea de acrecentar fuerzas frente a la posibilidad de invasiones por parte de España o Francia; esto, siguiendo la lógica de "El enemigo de mi enemigo, es mi amigo".

Las intenciones de México al incorporar a Centroamérica significaron, por un lado, una defensa militar ante una invasión europea y, por el otro, la oportunidad de la élite criolla de liberarse del poder político y comercial de Guatemala. Además, un aspecto importante de enfatizar es que la independencia de Centroamérica no fue emancipatoria, por lo que Ordoñez introduce el término de "equi-dependencia":

Centroamérica fue "víctima" no solo de la necesidad de México de tener respaldo militar para la defensa del imperio, sino del choque ideológico posrevolucionario de Francia y Estados Unidos. Por lo tanto, la separación de España no significó una in-dependencia en el sentido emancipatorio de la palabra, sino más bien, propongo, tuvimos una equi-dependencia de la administración de las provincias de España hacia México. (Comunicación personal, 20 de agosto, 2020)

En este caso, se alude a que la región solo cambia de dependencia, porque la "in-dependencia" que se dio de España no significó un corte de relaciones, sino una especie de una igual relación de dependencia trasladada para México y, con ello, las cuestiones políticas y relaciones comerciales.

Otro dato por destacar, trascendentalmente, es a quién interesaba la independencia de Centroamérica, ya que, más allá de ser integracionista, esta fue desde sus inicios, un movimiento criollo, que no buscó más que sus propios beneficios y la continuación de estos, puesto que, en ningún momento, tenía como objetivo la mejora de la vida de los propios centroamericanos. Con ello, se retoman y se fundamentan las líneas de análisis de la geopolítica crítica, en cuanto a "manipular", "conducir", "alinear", dado que desde los movimientos elitistas se procuraban y se dieron estos discursos de independencia. Así, Ordoñez establece que "La independencia fue un movimiento elitista, promovido por comerciantes de Guatemala y El Salvador para enriquecerse con los nuevos lazos comerciales que esperaban tener con Inglaterra, Francia, Holanda, el entonces Imperio Ruso y los Estados Unidos" (Ordoñez, comunicación personal, 2020). 
Entre otros factores internos, cabe destacar que la emancipación política de Centroamérica fue la primera expresión del regionalismo en ese trance, aunada a los intereses de las élites que se terminaron imponiendo sobre la visión de la América Central como región. También, debido a que la independencia llegó de manera diferenciada a cada uno de los países, principalmente a los de la parte sur de la región, el historiador José Cal señala que "es importante entender el espacio geográfico y que, a pesar de que las circunscripciones geçográficas desde la gran crisis final del Imperio Español hasta la emancipación política se mantienen, se cree que Centroamérica está destinada a estar unida, y eso es un error" (comunicación personal, 20 de agosto, 2020).

Desde una perspectiva de larga duración, hay fuerzas políticas y económicas que propiciaron momentos de integración en América Central, como el proyecto federal, pero igual de fragmentación. Con todo lo anterior, se puede concluir que Centroamérica ha heredado un autoritarismo político, lo que caracteriza a cada una de las naciones de la zona como Estado inestable y débilmente construido, que prevalece hasta nuestros días; a pesar de estar en una misma región y con una historia en común, parece ser que Centroamérica está destinada a no estar unida.

Tal como se ha señalado, la región centroamericana está condenada a rodearse de potencias y a que estas ejerzan influencia sobre ella, dada su posición geográfica y su valor para los actores del sistema internacional. Es así que, en el territorio centroamericano, se ha suscitado una serie de conflictos disfrazados como "problemas internos" que la han debilitado y han permitido que las potencias con mayor influencia puedan ejercer control sobre su territorio, sin mayor complicación.

\section{La región como espacio-ruta geopolítico a la disposición de las potencias}

Sobre la concepción de ruta, Ostos (2020) advierte que se trata de un "un objeto tanto geográfico como político, ya que es creadora y generadora de acceso" ( $\mathrm{p}$. 70). Por ello, Centroamérica se configura como un terreno estratégico, lo cual la dota de un espacio de poder geopolítico, generado por sus riquezas naturales, al igual que por su posición entre dos océanos, los cuales la perfilan como un puente terrestre y marítimo que facilita, principalmente, la rutas para el comercio.

También, Centroamérica se configuró como un espacio-ruta, estratégico y de interconexión, convirtiéndose en un punto clave para las potencias antagónicas de las pretensiones de Estados Unidos: la Federación Rusa, la República 
Popular de China, los países miembros de la Unión Europea, entre otros, que poco a poco han indo insertándose en el territorio centroamericano.

El vecino país del norte, a partir de los años noventa, se alejó un tanto de la región, debido a que dejó de ser parte de sus principales intereses; asimismo ocurrió tanto con la disminución de los aportes como con la ayuda económica por parte de la comunidad internacional, en comparación con lo recibido en tiempos de conflicto. Con el "estallamiento de la paz y al establecerse los regímenes democráticos en todos los países centroamericanos, parecía abrirse una era de creciente desarrollo económico, ya que la región regresaba a cierta normalidad" (Barrios, 2014, p. 199). Pese a los pronósticos, contradictoriamente, los resultados del cumplimiento de los Acuerdos de Paz no fueron los esperados en el área centroamericana, por lo que la pobreza siguió prevaleciendo y los aportes que se tenían por parte de cooperación internacional empezaban a resentirse en la sociedad; sin embargo, los fenómenos naturales se tornaron, cada vez más, una amenaza de interés y, de esta forma, la cooperación internacional volvió a llegar a Centroamérica.

En ese sentido, se puede observar cómo los intereses de las potencias han estado presentes y han marcado a Centroamérica; es por ello que, al analizar estas acciones desde la geopolítica crítica, es necesario recurrir al concepto de hegemonía, sobre el cual Agathangelou y Ling (2004) mencionan:

Donde ellos ven a Imperio como seguro, universalizable, y determinado, a cargo de un sistema capitalista de autorregulación, nosotros presentamos al imperio como una formación socio-política que intenta dos objetivos al mismo tiempo: 1) establecerse como la única posibilidad disponible para todos, y 2) negar, rechazar o borrar otros mundos. Hegemonía no es sólo una colusión entre las instituciones estatales y la sociedad civil para preservar los intereses de la élite, como Gramsci teorizó (...), pero también como esfuerzo sistemático para acabar con todas las otras formas de ver, hacer, ser y relacionarse en el mundo (p. 524).

Parece que dentro de la región centroamericana, a lo largo de la historia, los países - aunque comparten un mismo espacio, presentan problemas similares y tienen una historia en común - han estado destinados a no permanecer integrados para conformar la región; más bien, las potencias han visto en cada uno de los estados centroamericanos (individualmente) ciertas "virtudes" o espacios para poder insertarse y, como mencionan Agathangelou y Ling (2004), "establecerse como la única posibilidad disponible para estos países"( p. 524). 
En ese sentido, es conveniente resaltar, de manera muy sucinta, las características que presentan las naciones centroamericanas:

Panamá como plataforma de un esquema de control continental y el Canal de Panamá; Belice y Guatemala, en tanto son el flanco sur del T-MEC y el paso final de frontera para la droga y migraciones. Honduras, después del golpe contra Manuel Zelaya se afirma el control de Estados Unidos, mientras que Nicaragua se encuentra en rebelión con Estados Unidos y se posiciona como socio de Venezuela, mientras que Costa Rica, en constante debate sobre la militarización de las luchas antidrogas. Aunado a eso, se puede mencionar la relación Guatemala, El Salvador y Honduras como los países del Triángulo Norte de Centroamérica, espacio para el Plan Alianza para la Prosperidad (Barrios, 2014, p. 200).

Centroamérica se presenta, entonces, como una zona de alto tránsito y de influencia de EE. UU., con economías financiarizadas, dinámicas de expulsión masiva de personas y una creciente proyección de potencias extrarregionales mediante cooperación económica (soft-balancing). Así, el territorio centroamericano es sensible a los intereses estadounidenses: con alta asimetría y dependencia en materia comercial, necesidad de exteriorizar la seguridad fronteriza y el creciente imperativo de reducir la influencia de China y Rusia.

\section{¿Es posible pensar en una Centroamérica deconstruida?}

Para comprender la historia de la región centroamericana a las puertas del bicentenario de independencia, surgen iniciativas orientadas a la deconstrucción de los "discursos de victoria" asumidos por quienes integraron lo que O'Tuathail (1998) denominó la "geopolítica de los expertos". Para Centroamérica, estos discursos de victoria se observan únicamente en dos momentos: cuando se logra la emancipación del Reino de España y una vez que se independiza de la Federación Mexicana.

Como se ha explicado, si bien es cierto que uno de los enfoques centrales desarrollados por la geopolítica crítica conlleva el alinear, conducir, manipular y direccionar la conducta social, particularmente, estos se han centrado en identificar la manera con la que se construyen los discursos geopolíticos que sustentan las determinadas espacialidades. Cada uno de los componentes y procedimientos es encargado de naturalizar representaciones específicas y prácticas en torno a un espacio (geográfico-de poder) establecido. Así, vale la pena destacar que dichos discursos son conceptualizados como "capacidades específicas de 
los actores para construir significados acerca del mundo y sus actividades, mediante recursos socio-culturales" (Cairo, 2005), en este caso particular, aquellos vinculados al poder, espacio y lenguaje. En esa línea, a partir de los discursos nombrados, se puede comprender lo que Foucault (2002) denomina "el proceso de redefinición de las representaciones dominantes que configuran el imaginario" (p. 11), específicamente para Centroamérica.

Para lo previo, es imprescindible resaltar que un discurso geopolítico está fundamentado en:

una relación dialéctica y, finalmente, sintética o complementaria entre las representaciones del espacio y las prácticas espaciales. Dentro de esta relación dialéctica, se encuentran los códigos, signos y entendimientos que crean las condiciones necesarias para que pueda existir un uso, diseño, y explotación tanto del espacio como de los elementos activos que lo componen. Por su parte, las prácticas espaciales se refieren al ejercicio efectivo que se practica en los lugares y los conjuntos espaciales previamente interrelacionados, impuestos y organizados para la producción económica y la reproducción social (Cairo, 2005, p. 12).

En el escenario real del espacio centroamericano, las prácticas aludidas conllevan "el sostenimiento de las representaciones espaciales (de poder) que se normalizan a partir de la explotación de los recursos naturales, de la mano de obra barata de los emigrantes documentados e indocumentados, el tráfico de estupefacientes y drogas ilegales, etc." (Preciado y Uc, 2010, p. 69).

La independencia pretendía, entre muchos aspectos, reflejar la implantación de la sociedad moderna proclamada en la Revolución francesa, desde la visión liberal que exigía como premisa erradicar el antiguo régimen colonial: lo que era nuevo, lo republicano, la eliminación del sistema colonial anárquico. Esto implicaba la reorganización total del Estado, separándolo de la Iglesia y, por supuesto, retirándole el poder y la influencia que ejercía. El principio rector de la sociedad debía ser la igualdad ante la ley.

Por otra parte, como menciona Pinto Soria (1993), “en el ideario político del grupo conservador suponía la continuidad del orden colonial. Que el sistema de poder se mantuviera, como lo reflejaba la alianza entre la Corona y el grupo oligarca guatemalteco" (p.15). Esto suponía un cambio únicamente en quien ostentaba el poder, por lo que se le daba esa absoluta autoridad al grupo criollo emancipado de España; sin embargo, se buscaba el mantenimiento del régimen colonial heredado, en el cual la Iglesia seguía teniendo un papel clave como fuente de ideología y en la configuración del Estado y la sociedad. 
Este discurso, reflejo de ese microespacio de poder de los criollos en la región, permitía la naturalización artificial de identidades homogéneas, donde se supone que "habrá ricos y pobres, ignorantes y sabios porque en el sistema de las sociedades es difícil y acaso imposible distribuir las fortunas y dividir las luces con igualdad absoluta. Pero el pobre y el millonario; el ignorante y el sabio serán iguales ante la ley... por consiguiente, el dualismo colonial de indígenas y ladinos debía ser abolido" (Pinto Soria, 1993, p. 18), aunque en la práctica, y doscientos años después, se proyecte todo lo contrario.

En la geopolítica crítica, también es importante retomar los aportes que hace O’Tuathail respecto a la "geopolítica desde arriba" (geopolitics from above). Hace alusión a los análisis amparados bajo un discurso de poder, elitista y alejado de la realidad, que subyace en un ámbito social en general (O'Tuathail, 1998, p. 3); se interpreta así cómo los discursos de victoria en Centroamérica se dan desde arriba, desde su seno elitista criollo, no para liberar a los pueblos centroamericanos, sino como un cambio en el control de la región.

El estudio de la geopolítica crítica implica un abordaje desde una perspectiva decolonial desprendida de las representaciones dominantes y de una geopolítica reducida a los espacios, con el fin de interpretar esa conformación de contradiscursos críticos de las expresiones históricas de colonialidad (Preciado y Uc, 2010, p. 72).

Como bien menciona O'Tuathail (1998), al deconstruir una realidad ya existente, se logra establecer una nueva mirada al objeto de estudio. Desde esta aproximación, Centroamérica se dejaría de ver superficialmente, a cambio de introducirse en las entrañas mismas de un espacio geográfico; lo que puede ofrecer, más allá de lo evidente, se hace con el propósito de tener una mirada endógena de la región, que no se base en las descripciones realizadas desde afuera para trazar políticas nacionales y regionales, sino que sea mediante la propia mirada del istmo.

A partir de esa deconstrucción planteada, se pretende "trascender de las cuestiones puntuales y más allá de discursos o apariencias de lo geográfico, lo político, lo militar y lo socio-económico, para alcanzar una comprensión multidimensional del espacio geográfico en cuestión" (Ostos, 2020, p. 66). Además, al realizar este ejercicio, se podría identificar otras dinámicas relevantes vinculadas con aspectos "ecológicos, religiosos, culturales, temas de género, identificación de grupos vulnerables, población migrante y la presencia de organizaciones delictivas, así como otros aspectos que son necesarios en un análisis interpretativo desde la geopolítica crítica" (Villamizar, 2020, p. 69). No obstante, no se puede 
dejar de lado la historia de la región y cómo las potencias han estado presentes desde la época colonial hasta la actualidad.

Además, mediante esta práctica, se puede identificar de modo puntual, a los actores y agentes, intra- y extraterritoriales, que convergen e intervienen en esta área geográfica $\mathrm{y}$, precisamente, dentro de esos microespacios de poder, como bien menciona Foucault, así también determinar cómo la presencia de estos puede influir en el desarrollo de la región. Sin embargo, es posible afirmar la existencia de un común denominador en cuanto al establecimiento de las líneas que se dictan desde afuera hacia adentro de la región, frenando toda posibilidad de una autonomía regional, por lo que toda oportunidad de una deconstrucción de lo que hoy conocemos como Centroamérica quedaría en el imaginario social.

\section{Retos en los procesos integracionistas de la región}

El carácter interoceánico de la zona centroamericana dio la pauta para su desarrollo en los siglos XIX y XX. No obstante, con el tema de la globalización desde el siglo XXI, la región se convierte, tal y como lo indica Acuña (2016), "en un estrecho cuello entre dos masas continentales y un peaje entre dos océanos, constituyéndose así en un puente biológico, migratorio, comercial y cultural".

Si bien lo anterior se puede considerar un punto a favor que resalta su importancia geopolítica en el continente, también ha causado el interés del exterior por el control del istmo, hecho que ha influido en la falta de articulación de los países para lograr una completa integración. En el presente, Centroamérica se ha ido perfilando como un tablero, en el cual las diferentes potencias que emergen, conforme los años, han ido tomando un interés en ella. Allí ejercen control y evitan que una sola nación pueda tener hegemonía en el istmo; tal y como lo resaltan Pinto Soria (1993), y Granados Chaverri (1986): la región centroamericana se ha convertido en un potencial trampolín estratégico, en el cual se pueden fortalecer relaciones comerciales, culturales, tecnológicas y hasta militares, en cuanto a la situación con China, Estados Unidos, la Unión Europea e incluso México y Brasil.

Entre las nuevas perspectivas y enfoques globales, Centroamérica desempeña un rol fundamental. En esta línea, Acuña (2016) resalta: "es necesario reconocer que, a pesar de su condición periférica y subordinada, la dinámica interna del istmo ha estado siempre conectada a procesos globales o transnacionales, los cuales no solamente han padecido, sino que también sobre los cuales alguna influencia ha tenido". 
Con el fin de retomar los planteamientos de la geopolítica crítica, es necesario reflexionar sobre cómo deconstruir lo que actualmente se conoce como Centroamérica. Para ello, es necesario trascender las escalas locales y ubicarse en niveles regionales e inclusive en el ámbito global, entendiendo que la búsqueda de una zona homogenizada se torna un tanto complicada desde los distintos puntos de vista. Asimismo, se requiere no perder de vista su nexo con los distintos centros de poder mundial, desde la colonia hasta el presente.

Entonces, la idea es repensar a Centroamérica como un espacio de transferencias e interconexiones tanto económicas como sociales, pese a los dilemas y contradicciones que presentan los países. También, es importante destacar las fortalezas de la región, las cuales deben aprovecharse y consolidarse desde lo interno, para ir construyendo un área más fuerte en todos los ámbitos, con mayores posibilidades de posicionarse, con cierta autonomía, en el tablero geopolítico del que ha sido utilizada para beneficios externos por parte de las potencias; no se debe esperar a que las líneas y la solución a los problemas que presenta el territorio sean dictadas estrictamente desde afuera.

\section{Reflexiones finales}

A casi doscientos años de la conformación de los Estados centroamericanos, la región se enfrenta a problemas añejos y nuevos, tales como gobiernos autoritarios, racismo, dependencia, migraciones, vulnerabilidad ambiental, violaciones a los derechos humanos, entre muchos otros. Por ello, sería pertinente que las naciones del área trabajen en conjunto para la consecución y puesta de objetivos en común, con el objetivo de apostarle a mejorar tanto las condiciones como la calidad de vida de todos los habitantes y no esperar a que las potencias establezcan la hoja de ruta para resolver dichos conflictos.

El istmo se ha constituido como una plataforma en la cual las potencias han aprovechado sus características para la consecución de sus propios intereses; ejemplo de ello lo fue la anexión a México, la cual, en términos reales, fue utilizada como defensa del Imperio mexicano; la construcción del Canal panameño que conecta los dos océanos, o bien su condición de escenario exportador de revoluciones a finales del siglo XX.

Como los referentes centroamericanos y geopolíticos han señalado, en Centroamérica persisten dos elementos que hacen referencia a su situación pasada y actual: el primero es que la región es vista y analizada geopolíticamente desde afuera y que proyectar una organización espacial para ella es delimitada por potencias dominantes; el segundo, que el área está condicionada por los factores 
geopolíticos, en cuestiones del desarrollo económico y social. De esta manera, es que tanto los elementos geopolíticos como los socioeconómicos han estado intrínsecamente relacionados en el desarrollo (avances o retrocesos) del territorio.

Los países de la región centroamericana enfrentan complejidades comunes, muy propias y características, en las cuales se privilegia su condición y grado de desarrollo socioeconómico. Pese a lo anterior, esta parte central del continente continúa siendo de gran importancia geoestratégica, aspecto que debería considerarse en la búsqueda de mayores ventajas internas, específicamente, aquellas orientadas al hallazgo del bien común de sus habitantes. Es trascendental que en el marco del bicentenario de la independencia de Centroamérica se aborden estos temas críticamente, tomando en cuenta los discursos de poder y su influencia en la construcción de realidades, con el propósito de profundizar en las reflexiones históricas de la región y, a la vez, de contribuir con novedosos aportes tanto teóricos como metodológicos.

\section{Referencias}

Acuña, V. (2 de mayo de 2016). Somos un sendero geopolítico en el centro de América. El Faro. https://www.elfaro.net/es/206005/academico/18527/Somos-unsendero-geopolítico-en-el-centro-de-América.htm?st-full_text=all\&tpl=11

Agnew, J. (1998). Geopolitics: Re-visioning World Politics. Titivillus. https://www.academia.edu/18157456/ Agnew_John_Geopolitica_Una_Revision_De_La_Politica_Mundial

Agathangelou, A. y Ling, L. (2004). Power borders, security, wealth: lessons of violence and desire from september 11. International Studies Quartely, vol. 48 , núm. 3.

Baños, P. (2017). Así se domina el mundo. http://www.elespiadigital.com/index. $\mathrm{php} /$ bibliografia/libros-recomendados/19475-asi-se-domina-el-mundo?format $=$ pdf

Barrios, A. (2014). Narcoactividad y globalización. https://www.academia. edu/7375018/Los_desafíos_de_la_integración_regional_centroamericana_ un_intento_por_vislumbrar_las_perspectivas_futuras

BBC Mundo. (12 de diciembre de 2016). Centroamérica, la víctima "colateral" de la guerra contra el narcotráfico en México. BBC News. https://www.bbc.com/ mundo/noticias-america-latina-38233351

Cairo, H. (2005). "Prólogo. Re-pensando la Geopolítica: la renovación de ladisciplina y las aportaciones de John A. Agnew”, en J. Agnew: Geopolítica: Una revisión de la política mundial. Madrid: Trama Editorial. 
Cal, J. (20 agosto 2020). Reflexiones desde la geopolítica en el marco del Bicentenario. (Área de Estudios Internacionales, IIPS-ECP-USAC, Entrevistador) Comunicación personal.

CSUCA. (2014). Centroamérica: casa común e integración regional. W. Soto y M. Suárez (eds.). San José, Costa Rica. http://repositorio.csuca.org/23/1/centroamerica_integracion_regional_4.pdf

Foucault, M. (2002). Vigilar y castigar. Argentina: Siglo XXI Editores.

Foucault, M. (1980). Power/Knowledge: Selected Interviews and Other Writings 1972-1977. Nueva York: Colin Gordon /Pantheon.

González, L. (2017). Organización del espacio global en la geopolítica "clásica": una mirada desde la geopolítica crítica. Revista de Relaciones Internacionales, Estrategia y Seguridad. https://revistas.unimilitar.edu.co/index.php/ries/ article/view/2864/2798\#info

Granados, C. (1986). Geopolítica en Centroamérica. Cuadernos políticos, número 46. México D. F.: Ediciones Era.

Haushofer, K. (2013). Geopolítica del Océano Pacífico: estudios de las interrelaciones entre geografía e historia. PACs Verlag.

Kjellen, R. (2019). El Estado como forma de vida. Wentworth Press.Mahan, A. (1991). Mahan on Naval Strategy. (N. I. Press, Ed.) Annapolis. https://books. google.com.gt/books?id=BKIvBgAAQBAJ\&printsec $=$ frontcover\&source= gbs_ge_summary_r\&cad $=0 \# \mathrm{v}=$ onepage $\& \mathrm{q} \& \mathrm{f}=$ false

Molina, S. (2019). El contexto geopolítico del gobierno de Francisco Morazán. Diálogos rev. electr. hist. [online], vol. 20, n. 1, pp. 20-33. ISSN 1409469X. El contexto geopolítico en el pensamiento de Francisco Morazán (scielo.sa.cr)

Ordóñez, J. (20 agosto 2020) Reflexiones desde la geopolítica en el marco del Bicentenario. (Área de Estudios Internacionales, IIPS-ECP-USAC, Entrevistador) Comunicación personal.

Ostos, M. (2020). Guerras híbridas, intervencionismo y seguridad en la nueva concepción geopolítica de América Central. Guevara, G. (ed.), Geopolítica latinoamericana: mirando al mundo desde el sur (pp. 67-89). Bogotá Colombia: Universidad Externado de Colombia.

Ó Tuathail, G. (1998). De-Territorialized Threats and Global Dangers: Geopolitics, Risk Society, and Reflexive Modernization. Geopolitics 3, 17-31.

Palerm, A. y Wolf, E. (2008). Antropología y Marxismo. Universidad Autónoma Metropolitana, México D. F. https:/www.academia.edu/39321646/ Antropolog\%C3\%ADa_y_marxismo 
Pinto Soria, J. (1993). Historia General de Centroamérica. Tomo II: El Régimen Colonial. Madrid: Ediciones Siruela.

Preciado, J. y Uc. P. (2010). La construcción de una geopolítica crítica. CLACSO. vol. 1 , núm. 1 .

Ratzel, F. (1985). Geografía politica. Wentworth Press.

Ritter, C. (2001). Geografía general comparada. París: Adamant Media Corporation.

Torres-Rivas, E. (1985). Notas para comprender la crisis política internacional. En CECADE (ed.), Centroamérica: Crisis y Politica Internacional (tercera ed.). México: Siglo XXI Editores. https://books.google.com.gt/books?id=RXOrg ugSJP0C\&pg=PA39\&lpg=PA39\&dq=el+eslabon + mas + debil+edelberto + tor res\&source=bl\&ots $=$ ex2QznL1Uo\&sig=ACfU3U1yGNr14Th92kZ-mDF5d5 loduECmQ\&hl=es-419\&sa=X\&ved=2ahUKEwjbj7_2tNPsAhXJpFkKHZSI AVQQ6AEwEXoECAcQAg\# $\mathrm{v}=$ onepage $\& \mathrm{q}=\mathrm{el} \% 2$

United States Department of State. (2016). International Narcotics Control Strategy Report. Enforcement Affairs, Bureau for International Narcotics and Law.

Villacorta, J. (7 de mayo de 2019). China avanza en Centroamérica . Global Affairs Strategic Studies. Recuperado el 2020, de https://www.unav.edu/web/ global-affairs/detalle/-/blogs/china-avanza-en-centroamerica

Villamizar, F. (2020). Geopolitica latinoamericana: mirando al mundo desde el Sur. (G. d. Guevara, Ed.). Bogotá: Universidad. Recuperado el 2021, de https:/alacip.org/wp-content/uploads/2020/09/GUEVARA-Gisela-Ed..Geopolitica-latinamericana.pdf

Warf, B. (2006). Encyclopedia of human geography. London: Sage. 584 pp. 85 cloth. https://www.researchgate.net/publication/249872160_Book_review_Warf Beditor2006_Encyclopedia_of_human_geography_London_Sage_584_ pp_85_cloth_ISBN_978_0_76198_858_8_Douglas_I_Huggett_R_and Perkins_C_editors_2006_Companion_encyclopedia_of_geography_from_ 
